\title{
Three-dimensional diffractive micro- and nano-optical elements fabricated by electron-beam lithography
}

\author{
Ivan B. Divliansky*a ${ }^{*}$ Eric G. Johnson ${ }^{\mathrm{b}}$ \\ ${ }^{a}$ College of Optics and Photonics, University of Central Florida, Orlando, FL 32816 \\ ${ }^{b}$ Electrical and Computer Engineering, University of North Carolina Charlotte, Charlotte, NC 28223
}

\begin{abstract}
The broad development of the micro- and nano-technologies in the past few years increased the need of techniques capable of fabricating sub-micron structures with arbitrary surface profiles. Out of the several fabrication approaches (HEBS lithography, laser writing, etc.) the electron beam writing stands out as the one capable of the highest resolution, superior alignment accuracy and very small surface roughness. These characteristics make the technique greatly applicable in the fields of photonics and micro-opto-electro-mechanical-systems (MOEMS). Here we describe the specificity of fabricating 3D diffractive micro- and nano-optical elements using Leica EBPG 5000+ electron beam system. Parameters like speed of writing, dose accumulation, pattern writing specifics, etc. affect greatly the electronbeam resist properties and the desired $3 \mathrm{D}$ profile. We present data that can be used to better understand the different dependencies and therefore achieve better profile and surface roughness management. The results can be useful in future developments in the areas of integrated photonic circuits and MOEMS.
\end{abstract}

Keywords: electron-beam lithography, analog lithography, grey lithography, three-dimensional nano-fabrication, nanooptics, diffractive optics

\section{ELECTRON BEAM 3-D NANO-FABRICATION}

\subsection{Introduction}

Electron-beam writing has been developed for and is generally associated with binary writing in polymers achieved via exposing the resist with constant dose at the desired locations. If dose variation is implemented during the process of writing, the electron-beam resist accumulates different rates of exposure. Upon development this transforms into different resist thicknesses and correspondingly to three-dimensional profile of the final structure. The obtained nonplanar topography depends on the resist properties, dose distribution, proximity effect and specific machine parameters ${ }^{1,2}$. These parameters define entity which if theoretically developed can guarantee near perfect profile matching between the designed and the experimental structures. Most of the research in the area of fabricating continuous profiles via electron beam writing has been focused on developing profile prediction software which accounts for the proximity effect in combination with given dose distribution ${ }^{3}$. This paper will discuss machine related topics which can greatly influence the final results as well. Even that the study is related to a specific electron beam writer the conclusions can be applicable to other machines using the same operational principals.

For the experiments it was used a state-of-the-art electron beam system manufactured by Leica Microsystems (now Vistec Lithography), model EBPG 5000+. The system has Schottky emitter and is capable of operating at $20 \mathrm{kV}, 50 \mathrm{kV}$ or $100 \mathrm{kV}$ with a minimum spot size of less than $10 \mathrm{~nm}$. The three-dimensional diffractive optical structures written with the system demonstrated the need to better understand its operating deflection principles along with the beam parameterswriting speed dependence. In the following sub-sections these issues will be addressed in order to reveal the close connection between them and the experimental results obtained.

*ibd1@creol.ucf.edu; phone: 1 (407) 823 6827; fax: 1 (407) 823 6880; www.creol.ucf.edu

Micromachining Technology for Micro-Optics and Nano-Optics V and Microfabrication Process Technology XII, edited by Mary-Ann Maher, Harold D. Stewart, Jung-Chih Chiao, Thomas J. Suleski, Eric G. Johnson, Gregory P. Nordin, Proc. of SPIE Vol. 6462, 64621B, (2007) - 0277-786X/07/\$18 - doi: 10.1117/12.700999 


\subsection{Electron-beam lithography system Leica EBPG 5000+}

\subsubsection{Schottky emitter characteristics}

Schottky emitters have started to be widely used in most of the high-performance focused electron beam systems for SEM, TEM, Electron-beam lithography, and etc. These emitters outperform well the $\mathrm{LaB}_{6}$ and thermionic sources in source size and energy spread. They have as well less stringent requirements if compared to the cold-field emission sources. A distinct characteristic of the Schottky emitters is the high-brightness that can reach $5 \times 10^{8} \mathrm{~A} / \mathrm{cm}^{2} \mathrm{SR}$ which in comparison to $\mathrm{LaB}_{6}$ or tungsten is between one and two orders of magnitude higher ${ }^{4}$. Such brightness can be very valuable in case large currents are needed or can lead to heating problems as will be discussed in the next section of the paper.

\subsubsection{Deflection system and exposure strategy}

The instrument's general exposure strategy is based upon dividing the figures that will be written into large scan fields (also called main fields) that consist of smaller trapezium sub-fields ${ }^{5}$. The main fields correspond to the maximum beam deflection range and their size depends on the desired positioning grid resolution limited by the 16-bit digital-to-analog converter boards. To write in adjacent main field the machine uses the holder stage to translate the sample and if $5 \mathrm{~nm}$ position accuracy between two shapes is desired, the main field size will be $5 \mathrm{~nm} \mathrm{x} 2^{16}=327.68 \mu \mathrm{m}$. In this way the beam can be positioned anywhere in the main field with $5 \mathrm{~nm}$ accuracy. Deflecting an electron beam across main fields that are hundreds of microns in size is associated with big electric or magnetic coils and correspondingly with big settling times due to the larger fields that need to be applied, which at the end slows the writing process. In order to make the writing faster an additional sub-division of the pattern into trapezium sub-fields is implemented in the system. These fields are approximately two orders of magnitude smaller and inside them the electron beam is rastered at very high speed by another set of coils. These coils are a lot smaller due to the small deflection they need to exert and thus their settling times are also much smaller if compared to the main field ones. Such combination of main and trapezium fields allows this particular system to write with speeds up to $25 \mathrm{MHz}$ (company guarantees performance specification) and even up to $50 \mathrm{MHz}$ (with no performance specifications guaranteed). Figure 1 gives a graphical representation of the exposure strategy used by Leica EBPG 5000+. If in one main field there are areas which will not be written, the beam is deflected directly to the bottom left corner of the next figure so it does not loose time rastering with the beam off which is the case in pure raster systems. This is a second factor that helps decrease the total write time.

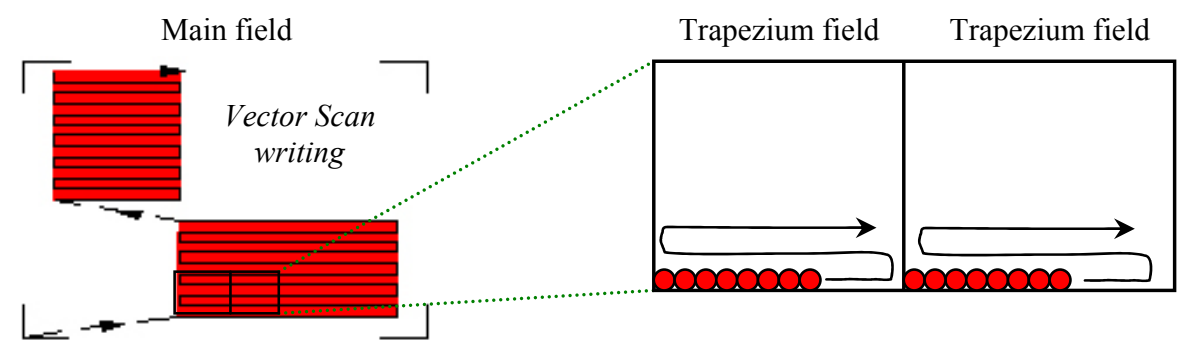

Fig. 1.Exposure strategy implemented in electron-beam writing system Leica EBPG 5000+

The size of the trapezium fields is governed by the user defined beam-step size and the file fracturing specifics, and can be adjusted if needed in the range of \pm 2048 bits. For given number of bits, such adjustment in units of length is trapezium size dependent. This is important to acknowledge because the already determined trapezium fields correction will be different when applied to differently fractured pattern or the same one but fractured with different beam-step size. There are several other characteristics of the system that are inter-related and important. The Leica EBPG 5000+ lithographic system is using a Gaussian beam to write point by point in a raster manner. In order to achieve a solid 
exposed line for example, the exposure points need to be close enough to each other so there is no area between them, unexposed or exposed with lower dose. The point separation called beam-step size (BSS) is user defined and totally dependant on the physical size of the chosen beam to use. It is accepted that a BSS smaller or equal to the diameter of the electron beam is enough to guarantee solid line exposures. The BSS affects the speed of writing as well which itself depends also on the desired resist dose and the beam current used to write. The formula defining the speed of writing in frequency units is given by equation (1), where $\boldsymbol{A}$ is the beam current and $\boldsymbol{D}$ is the exposure dose.

$$
F[M H z]=\frac{0.1^{*} A[n A]}{D\left[\frac{\mu C}{c m^{2}}\right] * B S S^{2}[\mu m]}
$$

The BSS appears as an important parameter being responsible for achieving smooth lines and also affecting the speed of writing.

\section{EXPERIMENTAL RESULTS AND DISCUSSION}

\subsection{Test patterns, dose distribution, and resist exposure}

Several test patterns were created to aid development of grey (analog) lithography using Leica EBPG 5000+ machine. The patterns represented basic optical structures with smooth or multi-level profile to guarantee better performance or improve a specific property. Our primary test geometry was a diffraction grating with lines consisting of 11 levels shown in Figure 2. In each period the dose was changed from 0.6 to 1.6 in steps of 0.1 around a set central dose. The lines with dose variation from 0.6 to 1.5 had widths of $500 \mathrm{~nm}$ and the last line was $2 \mu \mathrm{m}(1.6 *$ central dose $)$.

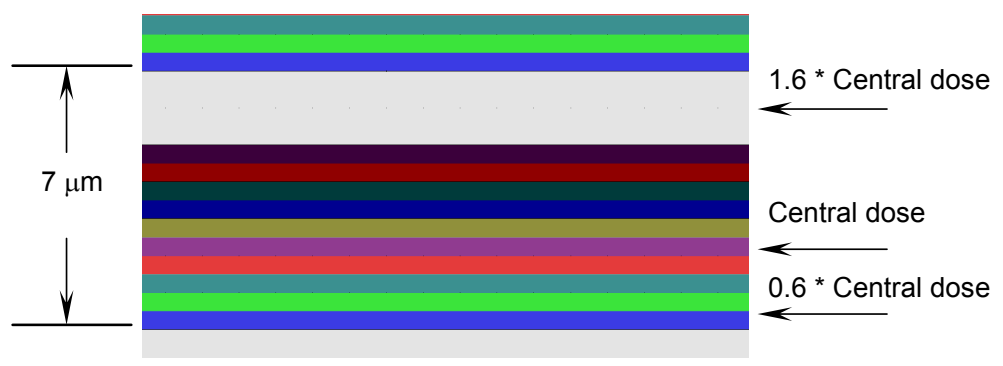

Fig. 2.Dose variation of multi-level diffraction grating pattern

Other test patterns included $1.2 \mu \mathrm{m}$ diffraction grating with 41 dose levels in every period, retroreflectors, and different sizes vortex-lenses. For our experiments we used PMMA 495 or ZEP 520 resists, deposited at 3000rpm for 50sec on a spin coater, and then baked at $180^{\circ} \mathrm{C}$ for 15 or $3 \mathrm{~min}$, correspondingly. After the exposure, the samples were developed in MIBK:IPA (1:3) or ZEP-RD for 75sec, rinsed in IPA for $10 \mathrm{sec}$ and then blow dried. The dose variation was automatically implemented by the machine so there was no need far manual adjustments or changes. To minimize the time of writing, the e-beam exploits a method in which a particular frequency/dose is written everywhere where it is present in the pattern before it switches to another one, and so on. This approach avoids many and fast changes between frequencies which improves the stability of the system. The relative line's positioning accuracy is guaranteed by a precision laser interferometer system governing the e-beam system stage movements. 


\subsection{Thermal effect}

The initial experiments with PMMA resist demonstrated two unexpected issues pictured in Figure 3. The test pattern exposed was the above mentioned $1.2 \mu \mathrm{m}$ diffraction grating with 41 dose variations per period. The SEM micrographs revealed non-uniformly exposed resist and a periodic under-exposed line pattern (white short lines perpendicular to the grating lines). In this sub-section we will focus on the former problem and will discuss the later in the following section.
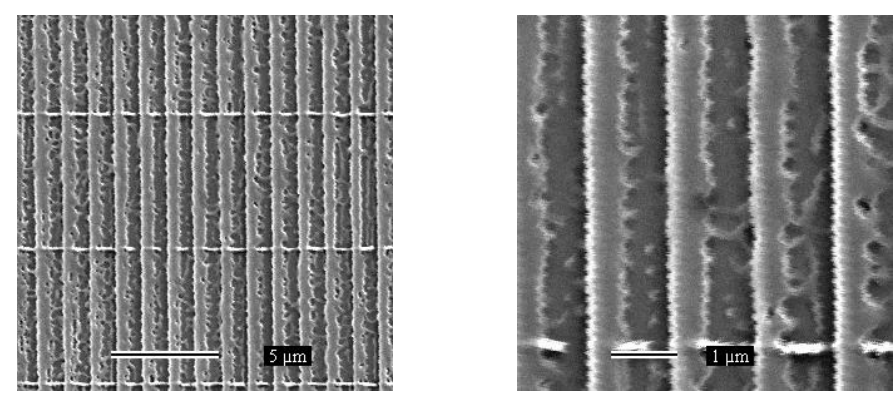

Fig. 3.Blazed diffraction grating exposed in PMMA with 41 dose variations per period

The developed resist profiles looked very much like dendrites randomly placed in the regions of every period and especially where the dose was getting higher. This led to the conclusion that it is most likely a thermal effect which caused the polymer to develop in such manner. If the polymer is overheated during the exposure, its properties will change drastically. It can be expected that some parts of it, even though exposed with high enough dose to clear, will be insoluble in the developer. Other parts can develop in random fashion based on the heat propagation, distribution, and its affect on the polymer. This coincides very well with the SEM micrographs showed in Figure 3. To further investigate and prove that there is thermal related issue an electron-beam resist with higher sensitivity was exposed with the same pattern. The resist used was ZEP 520 which needs approximately 4 times less dose to achieve the same depth profile at the same electron-beam accelerating voltage. The experiments confirmed that ZEP 520 is affected less but is still thermally damaged during the e-beam writing. In this case three additional points were considered as heat minimizing tools:

- use of smaller beam currents

- use of higher writing speeds

- $\quad$ multiple pass writing with fractional dose delivery

The implementation of smaller currents and higher writing speeds are two ways to lower the number of electrons per unit area, per unit time, reaching the sample and thus reducing the polymer heating. Considering equation (1) it is apparent that with lowering the beam current the speed of writing increases. In addition, the speed can be increased by using smaller BSS with lower limit governed by the $50 \mathrm{MHz}$ writing speed machine limit. If there is still a need to compensate for a residual thermal effect the resist dose could be delivered in several beam passes instead of one. For example, if a dose of $52.5 \mu \mathrm{C} / \mathrm{cm}^{2}$ is desired, it can be exposed in 7 passes each delivering a dose of $7.5 \mu \mathrm{C} / \mathrm{cm}^{2}$. This approach for writing is called "ghost" or "multiple-exposure" writing. Figure 4 shows a comparison between direct dose exposure (dose delivered in one pass) and ghost exposures of the grating described in Figure 2, done in ZEP resist. Each dose label in Figure 4 corresponds to the central dose of the exposure and the total dose spread is from 0.6 to 1.6 times the central one. The beam used to perform the exposures had current of 430pA and the beam-step size was set to $15 \mathrm{~nm}$. With minimum dose of $4.5 \mu \mathrm{C} / \mathrm{cm}^{2}\left(0.6 \times 7.5 \mu \mathrm{C} / \mathrm{cm}^{2}\right)$ the maximum writing frequency was approximately $48 \mathrm{MHz}$ and was close to the upper limit of the system. 

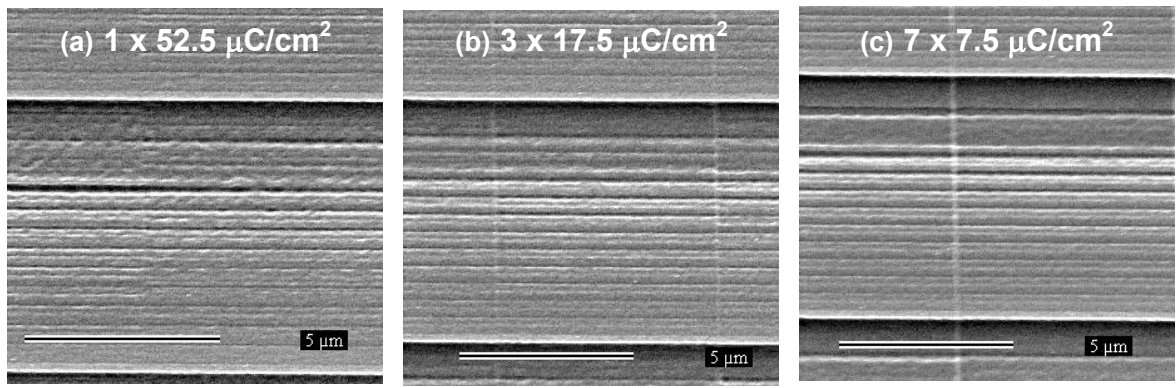

Fig. 4.Comparisson between direct dose writing (a) and ghost writing (b and c).

The SEM micrographs are presenting top view of a blazed diffraction grating written with 11 dose levels

Figure 4 demonstrates that ZEP is a lot less thermally affected if compared to PMMA even when the whole dose is delivered in one time $\left(1 \times 52.5 \mu \mathrm{C} / \mathrm{cm}^{2}\right)$. This is attributed to the lower exposure dose needed to achieve a comparative resist profile. In addition, implementing the ghost exposure technique led to substantial improvement of the surface quality of the written structures, and this is well demonstrated on Figures 4 (b) and (c). Applying the ghost writing effectively increased the speed (or frequency) of writing and delivered lower dose per single run. The results proved that implementing low currents, fast writing speeds and multi-pass dose delivery eliminated the initially observed thermal problem and achieved better surface smoothness. Each one of the heat-lowering methods mentioned above actually corresponded to lowering the number of electrons reaching the sample. Thus, the origin of the problem was associated with the high-brightness Schottky emitter present in the Leica electron-beam system.
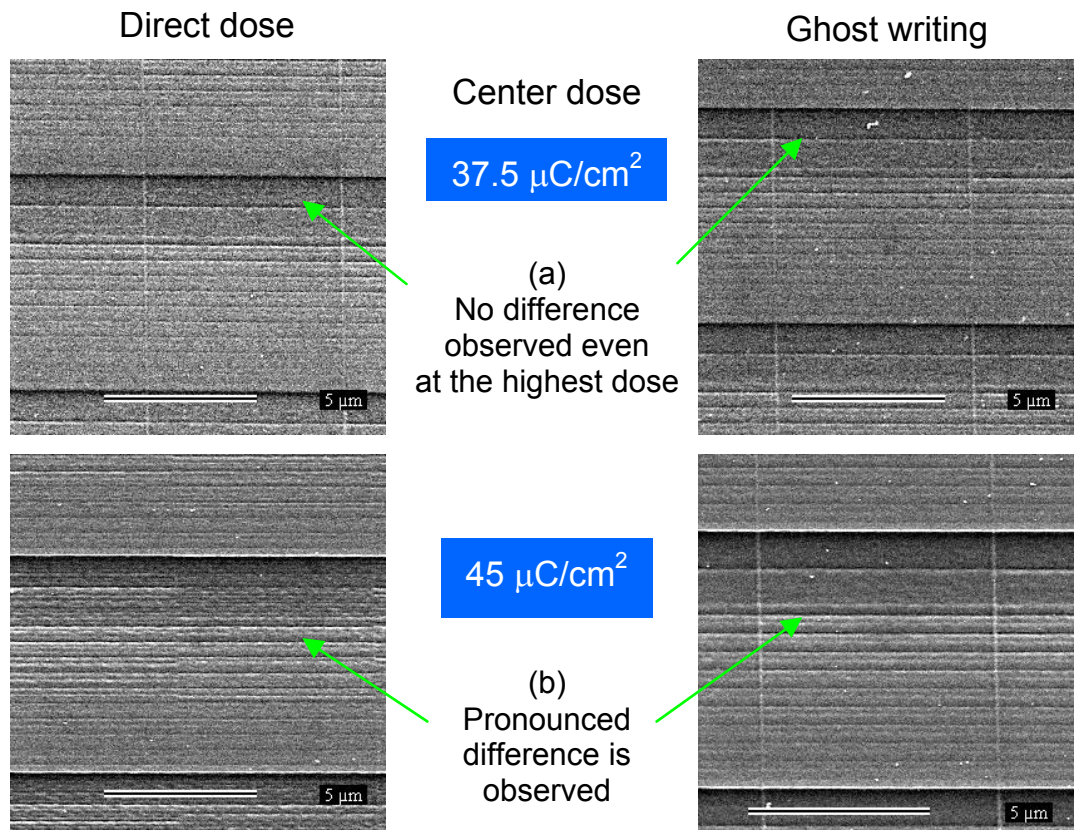

Fig. 5.Threshold behavior of the thermal effect observed upon multi dose resist exposure

As expected, the thermal problem showed to be a threshold effect and Figure 5 reveals this clearly. Upon implementation of below threshold dose direct dose writing no thermal related resist effect was observed and the results matched with the corresponding ghost writing sample (Figure 5a). When the dose exceeded the threshold a substantial surface quality difference was observed (Figure 5b). In conclusion, if the exposure dose range required is below the determined threshold, a direct dose writing can be used but in the cases where some or all doses exceed the threshold, ghost writing in combination with low-currents and fast writing speeds need to be implemented. 


\subsection{Trapezium boundaries compensation}

The second issue encountered in relation to writing analog structures with Leica EBPG 5000+ was the presence of trapezium boundaries (the white horizontal lines in Figure 3 and the vertical ones in Figures 4 and 5). As described earlier in this paper the electron beam system breaks every shape that needs to be written into main and trapezium fields. In order to move from main field to main field the machine is using a motorized interferometrically controlled stage, and to move from trapezium to trapezium it is deflecting the electron beam with a set of electric coils controlled by operational amplifiers. Even though the amplifiers can function up to $50 \mathrm{MHz}$ the maximum frequency guaranteed performance is limited to $25 \mathrm{MHz}$. At frequencies above $25 \mathrm{MHz}$ the amplifiers cannot follow up and they deflect the beam not to the full desired extent before it is moved to write the next line, thus a beam lag is introduced. This manifests itself as underexposure at the trapezium boundaries when positive resist is exposed. Figure 6 shows an example of ghostwritten blazed diffraction grating with a central dose of $7.5 \mu \mathrm{C} / \mathrm{cm}^{2}$ and different trapezium field sizes. The central dose corresponds to the highest speed of writing being $48 \mathrm{MHz}$. A well present trapezium boundary is observed when the default trapezium size is used at such high frequencies due to the amplifiers lag (Figure 6a). To eliminate the lag effect the trapezium fields need to be enlarged intentionally up to a size which will exactly compensate and cancel the boundary problem. The Leica EBPG 5000+ electron beam system can expand or shrink the trapezium size in the range of \pm 2048 bits, where the single bit physical size is proportional to the actual trapezium size. Figures $6 \mathrm{~b}$ and $\mathrm{c}$ demonstrate the level of underexposure when the fields are enlarged by 75 and 150 bits correspondingly. The 150 bits compensation is almost enough and the underexposed boundary is barely detectable. At 200 bits compensation an overexposure is observed.
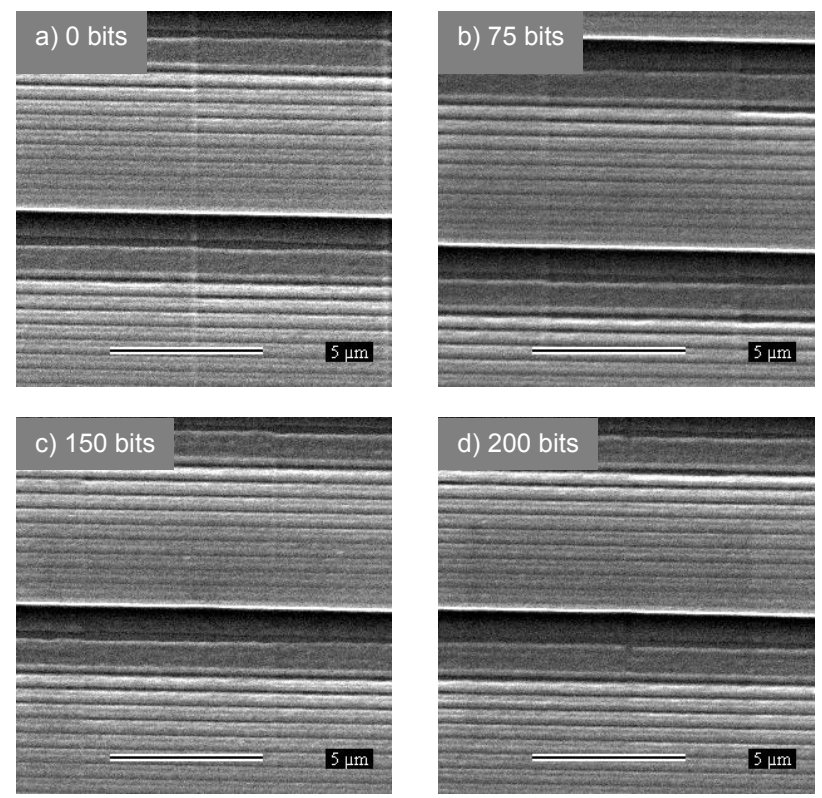

Fig. 6.Trapezium field size compensation applied to eliminate the presence of trapezium boundaries upon exposure at high frequencies

It needs to be acknowledged that due to the writing with different frequencies inside a given shape (for example - one grating period) and the frequency dependence of the beam deflection lag, the single trapezium size compensation may not work well. This will be very obvious if there is a great difference between the largest and smallest frequencies used to write an analog shape. For Leica EBPG 5000+ the span of frequencies is limited between 0.6 and 2 times the central frequency so careful planning and development of the dose-shape-trapezium size compensation dependence needs to be done to remove any overexposure lines for all the frequencies. 


\subsection{Examples of three-dimensional diffractive optical elements fabricated with Leica EBPG 5000+}

For demonstration purposes several different analog diffractive optical structures were designed and exposed in ZEP 520. Figure 7 shows a blazed diffraction grating exposed via ghost writing and trapezium size compensation of +180 bits. The dose was delivered through exposing 6 times the same pattern with central dose of $8 \mu \mathrm{C} / \mathrm{cm}^{2}$ using a $430 \mathrm{pA}$ beam. The second SEM image in Figure 7 also reveals that due to its high sensitivity ZEP is not quite the best polymer choice for analog writing. The uneven polymer present at the deepest part of every period is due to the electron scattering in combination with the abrupt dose change. Less sensitive polymers like PMMA should suffer a lot less from such effects and should give cleaner analog structures.
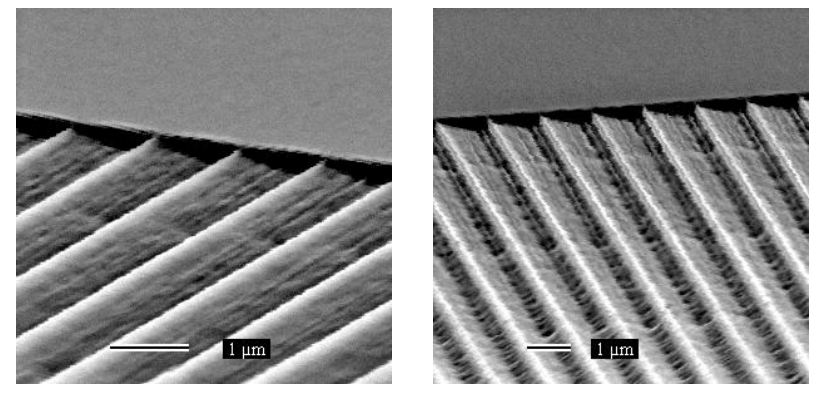

Fig. 7.Blazed diffraction grating with periodicity of $1.2 \mu \mathrm{m}$ written via ghost exposure $\left(6 \times 8 \mu \mathrm{C} / \mathrm{cm}^{2}\right)$ and +180 bits enlargement of the trapezium size

Figure 8 is an example of diffractive prisms with different sizes and heights written via the ghost exposure method. The images were taken with Zygo NewView 3D optical profiler and present a three-dimensional surface picture of the fabricated prisms. The heights of the prisms ranged from around $230 \mathrm{~nm}$ up to $1 \mu \mathrm{m}$ depending on the exposure dose used. A +180 bits trapezium boundary enlargement was used and the pictures show clearly the lack of any over or underexposed lines.
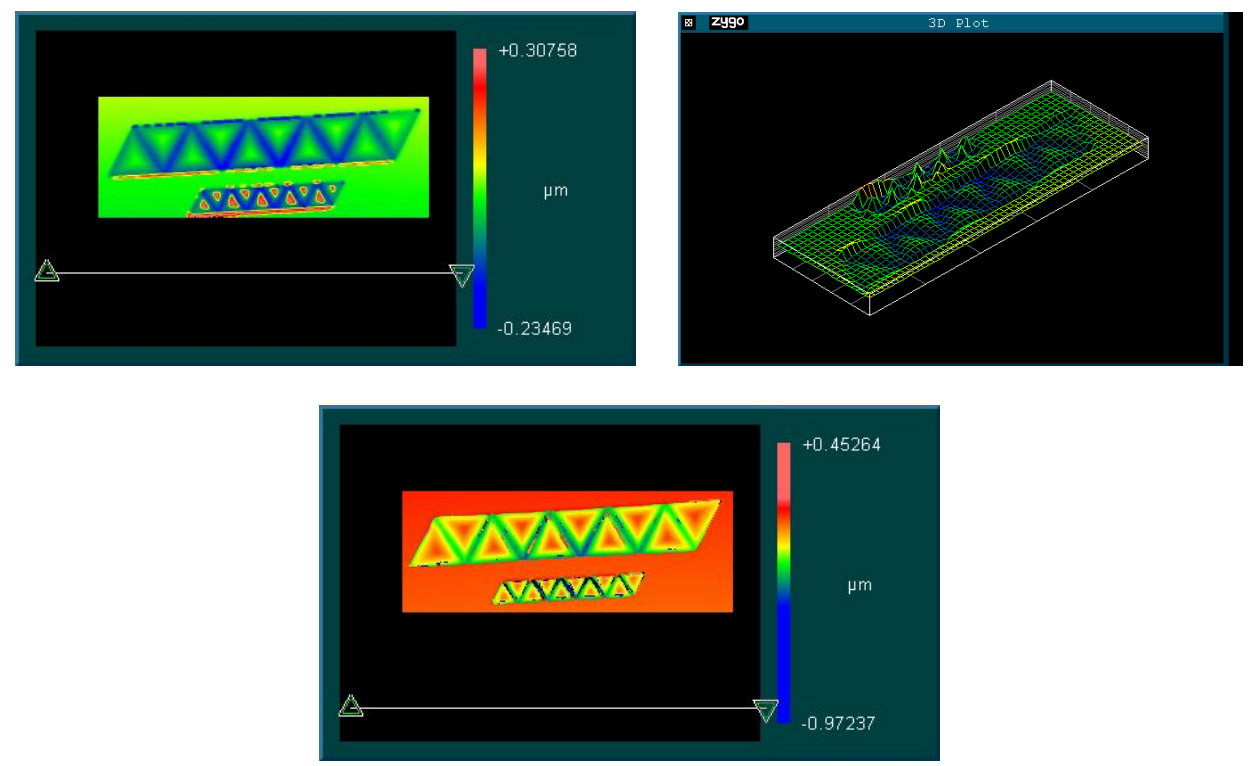

Fig. 8.Diffractive prisms with different heights written in ZEP 520 


\section{SUMMARY}

The growing interest in fabricating micro- and nano-structures with stepped or smooth 3D profiles led to the research presented in this paper. The experiments performed by using Leica EBPG 5000+ electron-beam writer revealed two major issues which were resolved in this study. First, randomly developed structures were observed and second, underexposed lines were present at the trapezium boundaries. The problem with the profile of the developed polymer was attributed to excessive heat accumulation. A combination of lower writing currents, faster writing speeds and multi-pass exposures proved the hypothesis that thermal changes in the polymer affected its properties and lead to undesired final structures. The origin of the thermal problem was associated with the high-brightness of the Schottky emitter source. The second issue being the presence of trapezium boundaries lines was a result of using writing frequencies above the specs of the operational amplifiers. At such speeds the amplifiers could not follow and deflect the electron beam to the full extent for each trapezium field. As a result the boundaries of the trapezium fields were underexposed. To solve the problem a correction was introduced which intentionally enlarged the size of the fields to the point where boundaries were not present. In conclusion, the results presented in the paper confirmed that machine related issues can lead to problems and close understanding of the electron-beam writer's principles can help to avoid and solve them.

\section{REFERENCES}

1. E.-B. Kley, "Continuous profile writing by electron and optical lithography", Microelectronic Engineering, 34, 261-298 (1997).

2. E.-B. Kley, B. Schnabel, U.D. Zeitner, "E-beam lithography - an efficient tool for the fabrication of diffractive and microoptical elements", SPIE 3008, 222-232 (1997).

3. M. Okano, H. Kikuta, Y. Hirai, K. Yamamoto and T. Yotsuya, "Optimization of diffraction grating profiles in fabrication by electron-beam lithography", Appl. Opt. 43(27), 5137-5142 (2004).

4. FEI Company, http://www.feibeamtech.com/pages/schottky.html

5. Leica EBPG 5000+ User's manual and Training notes. 